Compari son of the efficiencies of different af $f$ inity tags in the purification of a recombi nant secret ory prot ei $n$ expressed i $n$ si I kwor m I arval hemol ymph

\begin{tabular}{|c|c|}
\hline 著者 & $\begin{array}{l}\text { Doj i ma Takashi, N shi na Takuya, Kat o Tat suya, } \\
\text { Ueda H r oshi, Park Enoch Y. }\end{array}$ \\
\hline $\begin{array}{l}\text { journal or } \\
\text { publ i cat } i \text { on } t i t l e\end{array}$ & Bi ot echnol ogy and Bi oprocess Engi neer i ng \\
\hline vol une & 14 \\
\hline nunber & 3 \\
\hline page $r$ ange & 281- 287 \\
\hline year & 2009-07- 14 \\
\hline 出版者 & Spr i nger \\
\hline 権利 & $\begin{array}{l}\text { The fi nal publ i cat i on i s avai I abl e at Spr i nger } \\
\text { vi a } \\
\text { ht t p: //dx. doi . or g/10. 1007/s12257- 008 0258- } 2\end{array}$ \\
\hline URL & ht t p: //hdl . handl e. net /10297/9041 \\
\hline
\end{tabular}


(Title)

\section{Comparison of the Efficiencies of Different Affinity Tags in the Purification of a Recombinant Secretory Protein Expressed in Silkworm Larval Hemolymph}

(Authors)

Takashi Dojima, ${ }^{1}$ Takuya Nishina, ${ }^{2}$ Tatsuya Kato, ${ }^{2}$ Hiroshi Ueda, ${ }^{3}$ and Enoch Y. Park ${ }^{1,2 *}$

(Affiliations)

Laboratory of Biotechnology, Major in Bioscience, Graduate School of Science and Technology, Shizuoka University, 836 Ohya Suruga-ku, Shizuoka 422-8529, Japan, ${ }^{1}$ Laboratory of Biotechnology, Department of Applied Biological Chemistry, Faculty of Agriculture, Shizuoka University, 836 Ohya Suruga-ku, Shizuoka 422-8529, J apan, ${ }^{2}$ Department of Chemistry and Biotechnology, School of Engineering, University of Tokyo, Bunkyo-ku, Tokyo 113-8656, Japan. ${ }^{3}$

* Corresponding author

Tel: $+81-54-2384887$ Fax: $+81-54-2384887$

e-mail: yspark@agr.shizuoka.ac.jp 
Abstract Silkworms are useful bioreactors for heterologous protein expression when used in conjunction with the Bombyx mori nucleopolyhedrovirus (BmNPV) bacmid system. However, purification from silkworm hemolymph is difficult since it contains various kinds of proteins. In this study, we investigated an effective single-step method for the purification of affinity-tagged single-chain antibody variable region fragment (scFv) from silkworm larval hemolymph. A 5-fold higher expression level was obtained when ScFv was fused with the His tag than when it was fused with the Strep II or GST tags. However, the His tag was inadequate for single-step purification since it led to the nonspecific binding of contaminants. The purification recoveries of GST-, Strep II-, and His-tagged scFvs were $91.8 \%, 43.7 \%$, and $27.2 \%$, respectively. The specific amount of single-step purified GST-tagged ScFv was 2.2-2.7 fold higher than the amounts of the His- and Strep II-tagged constructs. The purities of Strep IIand GST-tagged ScFvs in the eluent were $98.4 \%$ and $83.0 \%$, respectively. Thus, both the short peptide Strep II and GST protein are suitable fusion tags for the affinity purification of proteins from silkworm larvae.

Keywords: silkworm larvae, Bombyx mori, antibody, affinity tag 


\section{NTRODUCTI ON}

Functional and structural analyses of proteins provide useful information but sufficient amounts of protein are required for these analyses. Therefore, large-scale purification of target proteins requires extensive research. Recombinant techniques are routinely used for sample preparation in pharmaceutical target screening as well as in functional and structural studies. These methods yield much larger amounts of protein than direct purification from source tissues. When recombinant proteins are fused with affinity tags, purification is relatively easier. In fact, approximately $75 \%$ of the proteins used in crystallization studies are expressed in the form of fusion proteins (2).

Recently, a great deal of attention has focused on the use of insect cells for protein expression since proteins can be secreted in these systems, leading to increased production of membrane proteins at the plasma membrane. Successful use of the baculovirus system for expressing integral membrane proteins for structural studies has been demonstrated (3). For example, several G-protein coupled receptors (GPCRs), such as types of dopamine, serotonin, muscarinic or adrenergic subtypes, have been successfully expressed in these systems (4).

Two major baculovirus expression systems have been developed, one based on Autographa californica multiple nucleopolyhedrovirus (AcMNPV) and the other based on Bombyx mori nucleopolyhedrovirus (BmNPV). The protein expression level is high when the BmNPV-silkworm larvae system is used. However, it had certain disadvantages about BmNPV since a considerable amount of time was required for the expression of the protein 
from the target gene. Nowadays this problem was cleared through the development of a bacmid (baculovirus shuttle vector) system for BmNPV (5). Since this method eliminates the need for multiple rounds of virus purification and amplification, it markedly reduces the technical difficulties and long times required for the selection and purification of recombinant viruses.

However, one problem faced during protein production in silkworm larvae is the purification of target proteins because the silkworm hemolymph contains various kinds of proteins. Therefore, it is necessary to perform several purification steps to prepare for protein sample of structural analysis, and which cause lower final recovery. If the purification yield is improved, the expression system using BmNPV-bacmid-based silkworm larvae could be more widely used. To overcome this difficulty, we attempted purification using an affinity tag that generally results in high yield. However, the efficiency of affinity purification may depend on the compatibility between the tag and host or between the tag and protein of interest. In the case of silkworm larvae, no details are available regarding the compatibility between the tag and protein of interest for efficient purification.

In this study, we investigated the efficiencies of different affinity tags for single-step purification of a target protein from silkworm larval hemolymph. Single chain antibody variable region fragment $(\mathrm{scFv})$ as a model protein was fused with various tags and expressed in silkworm larvae. Purity, recovery yield, and efficiency of single-step purification from silkworm larval hemolymph are discussed. 


\section{MATERI ALS AND METHODS}

\section{Silkworm larvae, strains, and plasmids}

Bombyx mori fifth-instar Fuyoutsukubane silkworm larvae (Ehime Sansyu, Yahatahama, Japan) were used in this study. The larvae were reared on an artificial diet (Silkmate 2S, Nihon Nosan, Yokohama, Japan) at $25^{\circ}$ C. E. coli DH5 $\alpha$, Gateway destination vector pDEST8, and TOPO pENTR vector were purchased from Invitrogen (Carlsbad, CA, USA). Plasmid pIT2-13CG2 (6), which encodes the human anti-bovine serum albumin (BSA) single-chain antibody variable region fragment ( $\mathrm{scFv}$ ), was kindly provided by Dr. Ian Tomlison of MRC Lab. of Mol. Biol. and MRC Center for Protein Engineering.

\section{Construction of recombinant BmNPV bacmids}

The affinity tags used for the purification of tagged scFv expressed in silkworm larval hemolymph were His, Strep II, and GST (Table 1).

The bacmid for the His-tagged 13CG2 fusion protein was constructed with N-terminus (His-13CG2 scFv) (Fig. 1A). A human scFv (13CG2) gene was amplified by PCR with pIT2-13CG2. The primers used were 13CG2-forward (5'-GAAGATCTGAGGTGCAGCTGTTGGAGTC-3') and 13CG2-reverse (5'-CTGGAATTCCCGTTTGATTTCCACCTTGGTC-3'). The amplified PCR product was 
purified using a GFX PCR purification kit (GE Healthcare) and digested with BglII and EcoRI. The resultant fragment was also purified and ligated into pBlueBacHis2 digested with BglII and EcoRI (pBBH-13CG2).

To secrete the expressed protein into the hemolymph of the silkworm, a bombyxin (bx) signal sequence (7) was added to 13CG2 scFv. Using pBBH-13CG2 as the template, PCR was carried out using the bx-His-forward (5'-CACCATGAAGATACTCCTTGCTATTGCATTAATGTTGTCAACAGTAATGTGGGTG TCAACACAACCGCGGGGTTCTCATCATC-3') and pBBH-reverse (5'-ACTTCAAGGAGAATTTCCTC-3') primers. The amplified DNA fragment was designated bx-His-13CG2 and purified using a GFX PCR purification kit.

The two DNA fragments were cloned into pENTR using pENTER/D-TOPO according to the protocol provided with the TOPO cloning system. The isolated plasmid pENTR/bx-His-13CG2 was cloned into pDEST8 using Gateway cloning technology (Invitrogen). pDEST8/bx-His-13CG2 was thus obtained. Transposition was carried out by transforming a donor plasmid pDEST/bx-His-13CG2 into E. coli Bm DH10Bac (5), which harbors the transposition helper plasmid pMON7124. The BmNPV bacmid/His-13CG2 was isolated on an LB plate containing $50 \mu \mathrm{g} / \mathrm{ml}$ kanamycin, $7 \mu \mathrm{g} / \mathrm{ml}$ gentamycin, $10 \mu \mathrm{g} / \mathrm{ml}$ tetracycline, $20 \mu \mathrm{g} / \mathrm{ml}$ chloramphenicol, $100 \mu \mathrm{g} / \mathrm{ml}$ 5-bromo-4-chloro-3-indolyl- $\beta$-D-galactopyranoside (X-gal), and $40 \mu \mathrm{g} / \mathrm{ml}$ isopropyl $\beta$-D-1-thiogalactopyranoside (IPTG).

The bacmid for Strep II-tagged scFv was constructed with the N-terminal His tag and 
C-terminal Strep II tag (Fig. 1B). The primers used for 13CG2 gene amplification were 13CG2-forward (5'-GAAGATCTGAGGTGCAGCTGTTGGAGTC-3') and 13CG2Strep-reverse

\section{(5'-CTGGAATTCCTTTTCGAATTGAGGGTGTGACCACCGTTTGATTTCCACCTTGGT}

C-3'). The amplified PCR product was purified as described above and digested with BglII and EcoRI. The resultant fragment was purified and ligated into pBlueBacHis2 digested with BglII and EcoRI (pBBH-13CG2-Strep).

The bombyxin (bx) signal sequence was added, using pBBH-13CG2-Strep as the template, PCR was carried out using the bx-His-forward

\section{(5'-CACCATGAAGATACTCCTTGCTATTGCATTAATGTTGTCAACAGTAATGTGGGTG} TCAACACAACCGCGGGGTTCTCATCATC-3') and 13CG2-reverse primers. The amplified DNA fragment was designated bx-His-13CG2-Strep and purified.

The two DNA fragments were cloned into pENTR using pENTER/D-TOPO as described above. The isolated plasmid pENTR/bx-His-13CG2-Strep was cloned into pDEST8, and pDEST8/bx-His-13CG2-Strep II was obtained. Transposition was carried out by transforming a donor plasmid pDEST/bx-His-13CG2-Strep into E. coli Bm DH10Bac as described as above, and BmNPV-His-13CG2 was obtained.

The bacmid for GST-tagged 13CG2 scFv was constructed with the N-terminal tandem His tag and GST tag (His-GST-13CG2 scFv), as shown in Fig. 1C. The glutathione S-transferase (GST) gene was amplified by PCR with pDEST20 using the Nhe I-GST-forward (5'-GATGCTAGCTCCCCTATACTAGGTTATTGG-3') and Nhe I-pGST-reverse 
(5'-GATGCTAGCACGCGGAACCAGATCCGAT-3') primers. The amplified GST fragment was digested with NheI and inserted into plasmid pDEST8/bx-His-13CG2 that was treated with NheI and calf intestine alkaline phosphatase and then screened for the correct orientation (pDEST8/bx-His-GST-13CG2).

The plasmid pDEST8/bx-His-GST-13CG2 was transformed into each E. coli Bm DH10Bac. The BmNPV bacmid was isolated using the same methodology as described above and used for the expression of the His-GST-13CG2 fusion protein.

\section{CG2 ScFv expression in silkworm larvae}

Forty micrograms of the rBmNPV bacmid DNA was suspended in $5 \mu$ of 1,2-dimyristyloxypropyl-3-dimethyl-hydroxy ethyl ammonium bromide (DMRIE)-C reagent (Invitrogen) and placed at room temperature for $45 \mathrm{~min}$. The resultant mixture was diluted to a final volume of $50 \mu \mathrm{l}$ with PBS, and $20 \mu \mathrm{l}$ of the bacmid mixture was then injected into the dorsum of the first day of fifth-instar silkworm larvae using a syringe with a 26-gauge beveled needle. The silkworm larvae were reared at $25^{\circ} \mathrm{C}$ in a breeding incubator. The infected larvae were harvested at 6 days post injection (d.p.i.), and the hemolymph was collected by cutting the caudal leg in a tube containing $5 \mu \mathrm{l}$ of $200 \mathrm{mM}$ 1-phenyl-2-thiourea; the hemolymph was centrifuged at $9000 \mathrm{rpm}$ for $10 \mathrm{~min}$ at $4^{\circ} \mathrm{C}$. The average amount of hemolymph obtained from each silkworm larva was $0.7 \mathrm{ml}$. The supernatant samples were immediately frozen at $-80^{\circ} \mathrm{C}$ for further analysis. 


\section{Purification of tagged 13CG2 scFv}

Two milliliters of hemolymph was filtered through a $0.45-\mu \mathrm{m}$ nitrocellulose membrane filter (Advantec, Japan) and diluted in $8 \mathrm{ml}$ of incubation buffer (conditioned sample). The conditioned sample was incubated with $1 \mathrm{ml}$ of the resin for $60 \mathrm{~min}$ at $4^{\circ} \mathrm{C}$ and then washed with 25 column volumes $(\mathrm{CV})$ of incubation buffer. The buffers used were as follows. For His tag incubation and washing, $20 \mathrm{mM}$ sodium phosphate (pH 7.8) with $0.5 \mathrm{M} \mathrm{NaCl}$ and $40 \mathrm{mM}$ imidazole was used. Elution was carried out with the same buffer containing different concentrations of imidazole, i.e., $100 \mathrm{mM}, 250 \mathrm{mM}$, and $500 \mathrm{mM}$. For Strep II tag incubation and washing, $100 \mathrm{mM}$ Tris- $\mathrm{HCl}(\mathrm{pH} 8.0)$ with $150 \mathrm{mM} \mathrm{NaCl}$ and $1 \mathrm{mM}$ EDTA was used. Elution was performed with the same buffer containing $2.5 \mathrm{mM}$ desthiobiotin. GST tag incubation and washing was carried out with $50 \mathrm{mM}$ phosphate $(\mathrm{pH} 7.3)$, while elution was performed with $50 \mathrm{mM}$ Tris- $\mathrm{HCl}(\mathrm{pH} 8.0)$ with $10 \mathrm{mM}$ reduced glutathione.

Elution was performed under gravity Ni Sepharose 6 FF, Strep Tactin Superflow agarose (Novagen), and Glutathione Sepharose 4 FF (GE Healthcare) affinity resins were used to purify the His-13CG2, His-13CG2-Strep, and His-GST-13CG2 fusion proteins, respectively.

\section{SDS-PAGE and western blot analysis}

The protein samples were analyzed by $12 \%$ sodium dodecyl sulfate-polyacrylamide gel 
electrophoresis (SDS-PAGE) using the Mini-Protean II system (Bio-Rad, Hercules, USA) and the Experion semiautomated electrophoresis system (Bio-Rad). The purity was determined with the Experion system by loading $4 \mu \mathrm{l}$ of denatured protein into a Pro $260 \mathrm{Chip}$, and the protein concentration was determined in accordance with the manufacturer's

recommendations. Western blot analysis was performed by electrophoretically transferring the SDS-PAGE gels onto polyvinylidine difluoride membranes using the TGM buffer ( $25 \mathrm{mM}$ Tris, $192 \mathrm{mM}$ glycine, 20\% methanol, $\mathrm{pH} \mathrm{8.3).} \mathrm{The} \mathrm{membrane} \mathrm{was} \mathrm{incubated} \mathrm{for} 1 \mathrm{~h}$ at room temperature in TBST (2.42 g/L Tris, $8 \mathrm{~g} / \mathrm{L} \mathrm{NaCl}$, and $1 \mathrm{ml} / \mathrm{L}$ Tween $20, \mathrm{pH}$ 7.6) containing 5\% skimmed milk powder. The anti-HIS G (Invitrogen) and anti-Strep antibodies were used at a dilution of 1:10000 in TBST. The anti-mouse horseradish peroxidase-conjugated antibody (GE Healthcare) was used as the secondary antibody at 1:20000 dilution. Detection was carried out using the ECL plus western blotting detection kit (GE Healthcare), and the bands were analyzed in a Fluor-S/MAX multi-imager (Bio-Rad).

\section{Determination of the protein concentration}

The protein concentration was determined using a Bradford protein assay kit (Bio-Rad).

\section{Enzyme-linked immunosorbent assay (ELISA)}

The amount of 13CG2 scFv in different samples was measured by ELISA (8). One hundred microlitter/well of $10 \mu \mathrm{g} / \mathrm{ml} \mathrm{BSA}$ was coated in a 96-well plates overnight at $4{ }^{\circ} \mathrm{C}$. A 
20\% skimmed milk solution in PBST (137 mM NaCl, $10 \mathrm{mM}$ phosphate, $2.7 \mathrm{mM} \mathrm{KCl}$, and $0.1 \%$ Tween 20, $\mathrm{pH} 7.4$ ) was added to the BSA-coated wells for blocking, for $1 \mathrm{~h}$ at room temperature. The wells were washed three times with the PBST buffer. The diluted samples were added to the well, incubated for $1.5 \mathrm{~h}$ at room temperature, and washed three times with PBST. The secondary antibody (Protein A-HRP diluted 1:1000 in 5\% skimmed milk/PBS) was added to each well (100 $\mu \mathrm{l} /$ well $)$ and incubated for $1 \mathrm{~h}$. After washing with PBST, the plates were incubated with $100 \mu \mathrm{l}$ substrate $\left(0.1 \mathrm{mg} / \mathrm{ml}: 3,3^{\prime}, 5,5^{\prime}\right.$ 'tetramethylbenzidine (TMBZ) in $100 \mathrm{mM}$ sodium acetate ( $\mathrm{pH} 6.0$ ), with $0.2 \%$ (v/v) of $30 \%$ hydrogen peroxide). The reaction was stopped by the addition of $50 \mu \mathrm{l}$ of $1 \mathrm{~N} \mathrm{H}_{2} \mathrm{SO}_{4}$ solution. The color that developed was measured at optical densities (OD) of $450 \mathrm{~nm}$ and $655 \mathrm{~nm}$ using a microplate reader (Model 680, Bio-Rad). The difference between $\mathrm{OD}_{655}$ and $\mathrm{OD}_{450}$ was used as a measure of the binding.

\section{RESULTS AND DISCUSSION}

\section{Construction of recombinant BmNPV bacmids and expression in silkworm larvae}

BmNPV bacmids/His-13CG2, His-13CG2-Strep, and His-GST-13CG2 were constructed for expressing scFv illustrated in Fig. 1. In the case of the bacmid with the Strep II tag, the antigen-binding activity of $\mathrm{scFv}$ was lost when the tag was inserted at the $\mathrm{N}$-terminus of scFv 
(data not shown). N-terminal placement of Strep II-tagged scFv (His-Strep-13CG2 scFv) might have partially masked the antigen-binding site.

The amounts of $\mathrm{scFv}$ in the larval hemolymph from the three bacmids were found to differ significantly, with the amount from His-13CG2 scFv being the highest $(150 \mu \mathrm{g} / \mathrm{ml})$ at 6 d.p.i., followed by His-13CG2-Strep scFv $(29.5 \mu \mathrm{g} / \mathrm{ml})$ and His-GST-13CG2 scFv $(8.5 \mu \mathrm{g} / \mathrm{ml})$ (Table 2). The specific amount of His-13CG2 scFv $(0.375 \mu \mathrm{g} / \mathrm{mg}$-protein) was similar to that of His-13CG2-Strep scFv (0.378 $\mu \mathrm{g} / \mathrm{mg}$-protein); however, the amount of His-GST-13CG2 $\operatorname{scFv}(0.12 \mu \mathrm{g} / \mathrm{mg}$-protein) was approximately one-third of these values (Table 2).

The S lanes in Figs. 2-4 show the expression pattern of the fusion protein in larval hemolymph. The bands of His-13CG2 scFv and His-13CG2-Strep scFv were of similar molecular size (30-35 kDa). In contrast, the His-GST-13CG2 scFv band (58 kDa) was confirmed to differ from the bands of the hemolymph proteins.

\section{Purification of affinity-tagged scFv from silkworm larval hemolymph}

Purification of His-tagged scFv The Ni Sepharose 6 FF column containing adsorbed His-13CG2 scFv was eluted with phosphate buffer using a stepwise imidazole gradient. The total elution volume was $3 \mathrm{ml}$, and this was collected in fractions of $1 \mathrm{ml}$ that were then subjected to protein analysis.

SDS-PAGE and western blotting analysis revealed a major band at $32 \mathrm{kDa}$ in the 
100-250 mM imidazole eluted fractions (Fig. 2). Several other contaminating proteins were also detected simultaneously. The amount of purified His-tagged scFv was $81.6 \mu \mathrm{g}$, as determined by ELISA. This was obtained by collecting and pooling 4 fractions purified from $2 \mathrm{ml}$ of hemolymph. The fold purification with respect to the hemolymph starting material was 736 . Single-step purification using the affinity tag resulted in a recovery yield of $27.2 \%$. These results suggest that the His tag may not be the best affinity tag for single-step purification of recombinant proteins from larval hemolymph.

Purification of Strep II-tagged scFv $\quad$ His-13CG2-Strep scFv was purified on a Strep Tactin Superflow agarose column eluted with $2.5 \mathrm{mM}$ desthiobiotin in Tris- $\mathrm{HCl}$ buffer.

SDS-PAGE and western blotting analyses revealed a major band at $35 \mathrm{kDa}$ (Fig. 3). Bands of higher molecular weights were only detected by western blotting using the anti-Strep antibody. This suggested either that western blotting is more sensitive than SDS-PAGE, or the possibility that the two scFv molecules associate to form a dimer.

Based on the ELISA results, $25.8 \mu \mathrm{g}$ of purified Strep II-tagged scFv was obtained from $2 \mathrm{ml}$ hemolymph. The fold purification with respect to the hemolymph starting material was 873. The recovery yield was $43.7 \%$.

Purification of GST-tagged scFv His-GST-13CG2 scFv was purified on a Glutathione Sepharose $4 \mathrm{FF}$ column eluted with $5 \mathrm{ml}$ of glutathione buffer ( $50 \mathrm{mM}$ Tris- $\mathrm{HCl}(\mathrm{pH} 8.0)$ and $10 \mathrm{mM}$ reduced glutathione), and 1-ml fractions were collected.

The major His-GST-13CG2 scFv band was observed at $58 \mathrm{kDa}$ (Fig. 4). Some lower molecular weight contaminants were also observed in the western blot, and these may be the 
degradation products of His-GST-13CG2 scFv.

Based on the ELISA results, $15.6 \mu \mathrm{g}$ of purified GST-tagged scFv was obtained from 2 $\mathrm{ml}$ hemolymph. The fold purification with respect to the hemolymph starting material was 6277. The single-step affinity purification resulted in a recovery yield of $91.8 \%$, indicating that most of the expressed protein was easily isolated on glutathione Sepharose. The specific amount of purified GST-tagged scFv was $747 \mu \mathrm{g} / \mathrm{mg}$-protein; this value is approximately 2.2-2.7 fold higher than the values obtained with the His and Strep II tags.

\section{Purity confirmation of affinity-tagged scFv}

To further confirm whether tagged scFv was assembled into dimers, each affinity purified eluent was analyzed with the Experion semiautomated electrophoresis system. Each scFv fused with the Strep tag and GST tag was eluted from the column in a single and defined peak

(Fig. 5). The purities of eluted His-13CG2-Strep and His-GST-13CG2 scFvs were $98.4 \%$ and 83.0\% respectively (Table 3 ). The molecular weights of His-13CG2-Strep and His-GST-13CG2 scFvs were shown as single peak and calculated to be $35 \mathrm{kDa}$ and $58 \mathrm{kDa}$, respectively, based on the molecular weight standards used.

From the Experion analysis, it was concluded that the Strep II tag was the most useful for target protein purification from silkworm larval hemolymph. 


\section{Evaluation of affinity purification in silkworm larval hemolymph}

In addition to purity and yield, cost is also a critical parameter when choosing affinity tags and resins (9). When the prices of different affinity resins are compared, it is observed that it costs $\$ 220$ to purify $1 \mathrm{mg}$ of a 32-kDa protein (based on the retail cost and resin capacity provided by the manufacturer). Strep-Tactin was the most expensive resin, since it costs $\$ 17,000$ to purify $1 \mathrm{mg}$ of the tagged protein. Glutathione Sepharose was moderately expensive since it costs $\$ 1,020$ to purify $1 \mathrm{mg}$ of the tagged protein (Table 3 ). The GST tag is widely used as a fusion tag and the target protein is generally fused to the C-terminus of GST. In general, the expressed target protein is removed from GST by using a specific protease that recognizes and cleaves the linker between the target protein and GST. However, there are certain problems in the cleavage step such as low yields, high costs, and nonspecific digestion, i.e., cleavage in regions other than the linker region. Despite these considerations, the crystal structures of fusion proteins containing large affinity tags have been recently reported (10-13). Several GST fusion proteins have been successfully used to drive the crystallization of target peptides and regulatory domains and to determine their structure (14). Therefore, the large GST tag was used in this study to purify the target protein sample from silkworm larval hemolymph.

\section{Conclusion}

We investigated the efficiencies of different affinity tags in single-step purification of recombinant proteins from larval hemolymph using the BmNPV bacmid expression system. 
The short Strep II peptide tag and the GST protein tag can be used for protein purification from silkworm larval hemolymph. The results of this study will be very useful in designing protocols for the purification of target proteins for structural analysis

Acknowledgment This study was supported by the Program of Basic Research Activities for Innovative Biosciences (PROBRAIN), Japan.

\section{REFERENCES}

[1] Derewenda, Z.S. (2004) The use of recombinant methods and molecular engineering in protein crystallization. Methods 34, 354-363.

[2] Kang, C.S., C.W. Park, I.S. Bang (2008) Production and purification of a cecropin family antibacterial peptide, hinnavin II, in Escherichia coli. Biotechnol. Bioprocess Eng. 13, 377-382.

[3] Madden, D.R. and M. Safferling (2007) Baculoviral expression of an integral membrane protein for structural studies. Methods Mol. Biol. 363, 39-57.

[4] Massotte, D. (2003) G protein-coupled receptor overexpression with the baculovirus-insect cell system: a tool for structural and functional studies. Biochim. Biophys. Acta 1610, 77-89.

[5] Motohashi, T., T. Shimojima, T. Fukagawa, K. Maenaka, E. Y. Park (2005) Efficient large-scale protein production of larvae and pupae of silkworm by Bombyx mori nuclear 
polyhedrosis virus bacmid system. Biochem. Biophys. Res. Commun. 326, 564-569.

[6] Aburatani, T., H. Ueda, T. Nagamune (2002) Importance of a CDR H3 basal residue in $\mathrm{V}(\mathrm{H}) / \mathrm{V}(\mathrm{L})$ interaction of human antibodies. J. Biochem. 132, 775-782 .

[7] Adachi, T., S. Takiya, Y. Suzuki, M. Iwami, A. Kawakami, S. Y. Takahashi, H. Ishizaki, H. Nagasawa, A. Suzuki (1989) cDNA structure and expression of bombyxin, an insulin-like brain secretory peptide of the silkmoth Bombyx mori. J. Biol. Chem. 264, 7681-7685.

[8] Ishikiriyama, M., T. Nishina, T. Kato, H. Ueda, E. Y. Park (2008) Human single-chain antibody expression in the hemolymph and fat body of silkworm larvae and pupae using BmNPV bacmids. J. Biosci. Bioeng. in press.

[9] Lichty, J.J., J. L. Malecki, H. D. Agnew, D. J. Michelson-Horowitz, S. Tan (2005) Comparison of affinity tags for protein purification. Protein Expr. Purif. 41, 98-105.

[10] Kobe, B., R. J. Center, B. E. Kemp, P. Poumbourios (1999) Crystal structure of human T cell leukemia virus type 1 gp21 ectodomain crystallized as a maltose-binding protein chimera reveals structural evolution of retroviral transmembrane proteins. Proc. Natl. Acad. Sci. 96, 4319-4324.

[11]Liu, Y., A. Manna, R. Li, W. E. Martin, R. C. Murphy, A. L. Cheung, G. Zhang (2001) Crystal structure of the SarR protein from Staphylococcus aureus. Proc. Natl. Acad. Sci. $98,6877-6882$.

[12]Ke, A., C. Wolberger (2003) Insights into binding cooperativity of MATa1/MATalpha2 from the crystal structure of a MATa1 homeodomain-maltose binding protein chimera. Protein Sci. 12, 306-3012.

[13]Cho, E.K. (2007) Enhanced tolerance against freezing stress in Escherichia coli cells 
expressing an algal cyclophilin gene. Biotechnol. Bioprocess Eng. 12, 502-507.

[14]Zhan, Y., X. Song, G. W. Zhou (2001) Structural analysis of regulatory protein domains using GST-fusion proteins. Gene 281, 1-9. 


\section{Figure legends}

Fig. 1. Schematic diagrams of the affinity-tagged scFv constructs of BmNPV bacmid/His-13CG2 (A), His-13CG2-Strep (B), and His-GST-13CG2 (C).

Fig. 2. SDS-PAGE (A) and western blot analysis (B) of His-tagged 13CG2 scFv purified on Ni Sepharose. Lane M: Molecular weight marker, A1-3: 100 mM imidazole eluant, B1-3: 250 mM imidazole eluant, C1-3: $500 \mathrm{mM}$ imidazole eluant, and S: hemolymph. Anti-HIS G was used in the western blot analyses. The arrow indicates the molecular weight of His-tagged $13 \mathrm{CG} 2 \mathrm{scFv}$.

Fig. 3. SDS-PAGE (A) and western blot analysis (B) of Strep II-tagged 13CG2 scFv purified on Strep Tactin Superflow agarose. Lane M: Molecular weight marker, F1-5: eluted fractions with $2.5 \mathrm{mM}$ desthiobiotin, S: hemolymph. Anti-Strep was used in the western blot analysis. The upper and lower arrows are supposed the monomers and dimers of Strep II-tagged 13CG2 scFv, respectively.

Fig. 4. SDS-PAGE (A) and western blot analysis (B) of GST-tagged 13CG2 scFv purified on glutathione Sepharose. Lane M: Molecular weight marker, F1-5: eluted fractions with $10 \mathrm{mM}$ reduced glutathione, and S: hemolymph. Anti-HIS G was used in the western blot analysis. The arrow indicates the molecular weight of GST-tagged 13CG2 scFv.

Fig. 5. Electrogram of purified Strep II- (A) and GST-tagged scFvs (B). The eluents obtained 
from the affinity purification columns were analyzed using the Experion electrophoresis system. The arrows indicate the peak of each purified protein. 
1 Table 1. Characteristic features of affinity-tags

\begin{tabular}{lccc}
\hline Tag & His-tag & Strep-tag (II) & GST-tag \\
\hline Size (residues) & $0.84 \mathrm{kDa}(6)$ & $1.1 \mathrm{kDa}(8)$ & $26 \mathrm{kDa}(211)$ \\
Sequence & HHHHHH & WSHPQFEK & Protein \\
Ligand & $\mathrm{Ni}^{2+}, \mathrm{Co}^{2+}$ & Strep-Tactin & Glutathione \\
Increase solubility & $\mathrm{No}$ & $\mathrm{No}$ & Yes \\
Tag position & $\mathrm{N}$ or C terminus & $\mathrm{N}$ or C terminus & $\mathrm{N}$ terminus \\
Elution agent & Imidazole & Desthiobiotin & Reduced glutathione \\
Capacity & $5-14 \mathrm{mg} / \mathrm{ml}$ & $50-100 \mathrm{nmol} / \mathrm{ml}$ & $10 \mathrm{mg} / \mathrm{ml}$ \\
\hline
\end{tabular}

2 
4 Table 2. Expression of $13 \mathrm{CG} 2 \mathrm{scFv}$ fusion protein in hemolymph

\begin{tabular}{lccc} 
Tag & His-tag & Strep-tag (II) & GST-tag \\
\hline $\operatorname{scFv}(\mu \mathrm{g} / \mathrm{ml}$-hemolymph) & 150 & 29.5 & 8.5 \\
$\operatorname{scFv}(\mu \mathrm{g} /$ larvae $)$ & 105 & 20.5 & 6.0 \\
Total protein $(\mathrm{mg} / \mathrm{ml})$ & 400 & 78.0 & 71.5 \\
$\mathrm{scFv} /$ total protein $(\mu \mathrm{g} / \mathrm{mg})$ & 0.375 & 0.378 & 0.119 \\
\hline
\end{tabular}

5 The amount of scFv was measured by ELISA.

6 
7 Table 3. Single-step purification of $13 \mathrm{CG} 2 \mathrm{scFv}$ fusion protein from hemolymph

\begin{tabular}{|c|c|c|c|}
\hline Tag & His - tag & Strep-tag (II) & GST-tag \\
\hline Hemolymph used (ml) & 2.0 & 2.0 & 2.0 \\
\hline Collection volume (ml) & 4.0 & 1.0 & 3.0 \\
\hline $\mathrm{scFv}(\mu \mathrm{g} / \mathrm{ml}-$ pool $)$ & 20.4 & 25.8 & 5.2 \\
\hline Total protein $(\mu \mathrm{g} / \mathrm{ml}-$ pool $)$ & 73.8 & 78.0 & 6.97 \\
\hline Amount of $\mathrm{ScFv}(\mu \mathrm{g})$ & 81.6 & 25.8 & 15.6 \\
\hline Amount of total protein $(\mu \mathrm{g})$ & 295.0 & 78.0 & 20.9 \\
\hline Specific amount of scFv $(\mu \mathrm{g} / \mathrm{mg})$ & 276 & 330 & 747 \\
\hline Purification recovery $(\%)$ & 27.2 & 43.7 & 91.8 \\
\hline Purification efficiency (fold) & 736 & 873 & 6,277 \\
\hline Purity by Experion $(\%)$ & NA & 98.4 & 83.0 \\
\hline Cost of gel $(\$ / \mathrm{ml})$ & 8.80 & 440.00 & 15.84 \\
\hline Gel price & $\$ 220(25 \mathrm{ml})$ & $\$ 11,000(25 \mathrm{ml})$ & $\$ 396(25 \mathrm{ml})$ \\
\hline Cost/mg-scFV (\$/mg) & 108 & 17,000 & 1,020 \\
\hline Commercial supplier & GE Healthcare & Novagen & GE Healthcare \\
\hline
\end{tabular}

$8 *$ His-tag and GST-tag were collected by 4 and 3 fractions respectively. 


\section{$9 \quad$ NA : Not analysis}

10 The capacity for the fusion protein adsorption and retail cost information obtained from manufacture's catalog. The unit cost was calculated 11 for purification of $1 \mathrm{mg}$ of a $32 \mathrm{kDa}$ protein fused to the appropriate tag, and the value of GST fusion take into account the large size of the 12 affinity-tag. 
(A) BmNPV bacmid/His-13CG2

$\mathrm{NH}_{2}$ Wh $\mathbf{V}_{\mathbf{H}} \mathbf{V}_{\mathbf{L}} \mathrm{COOH}$

(B) BmNPV bacmid/His-13CG2-Strep

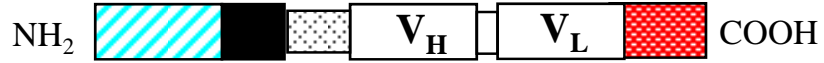

(C) BmNPV bacmid/His-GST-13CG2

$\mathrm{NH}_{2}$

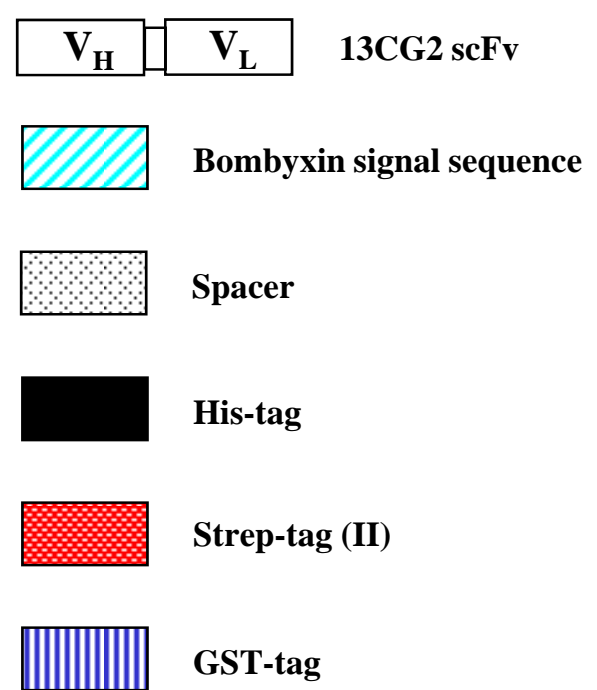


(A) SDS-PAGE

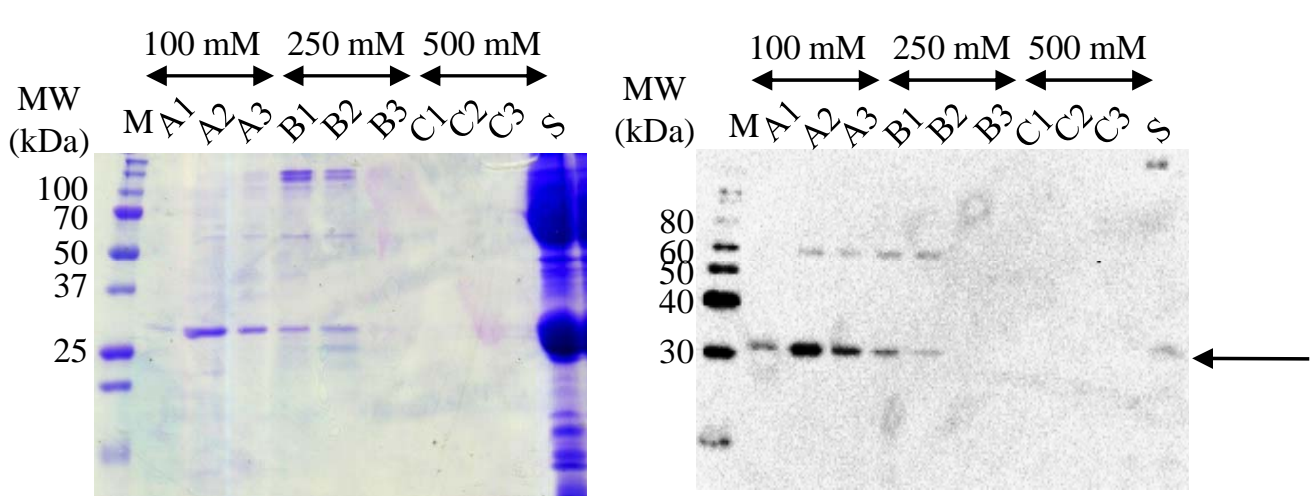




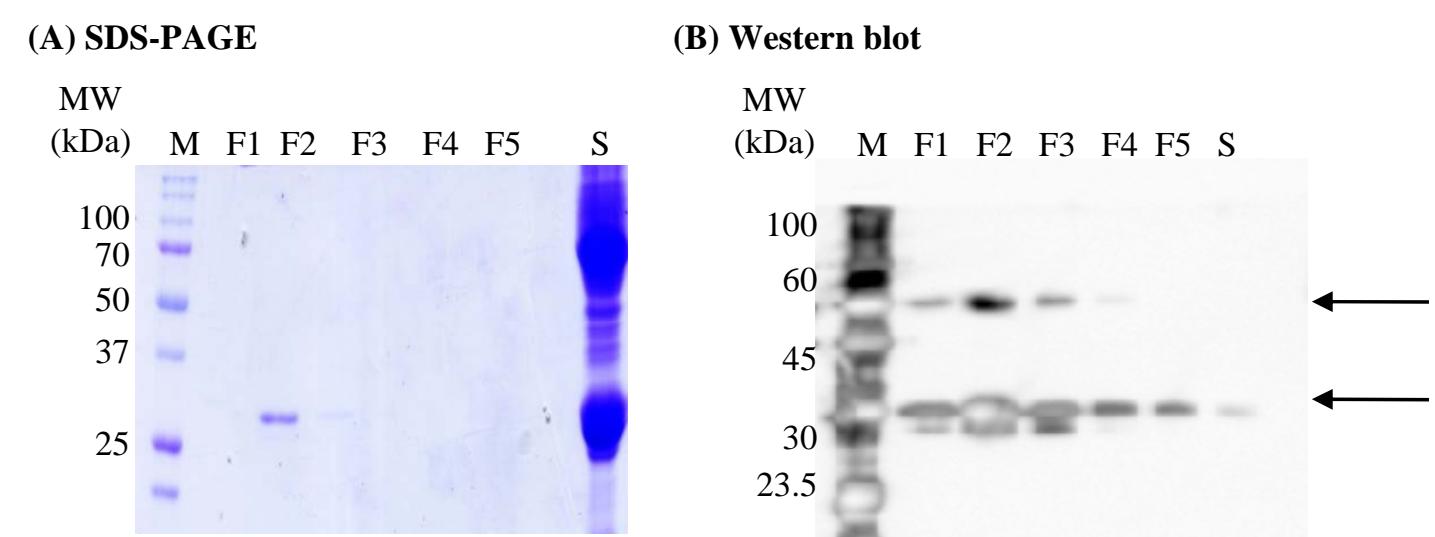




\section{(A) SDS-PAGE}

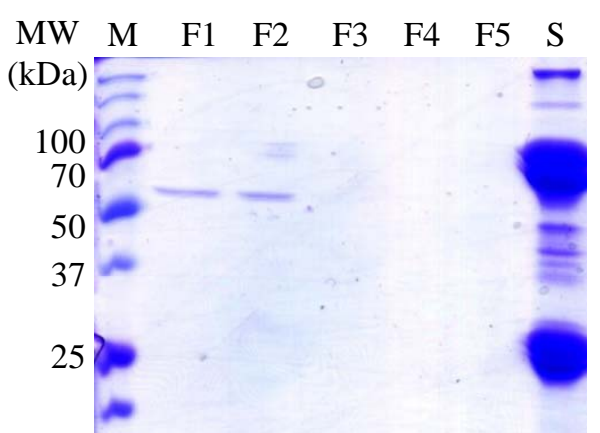

(B) Western blot

$\begin{array}{llllllll}\text { MW } & \text { M } & \text { F1 } & \text { F2 } & \text { F3 } & \text { F4 } & \text { F5 } & \text { S }\end{array}$ (kD)

60

50

40

30 

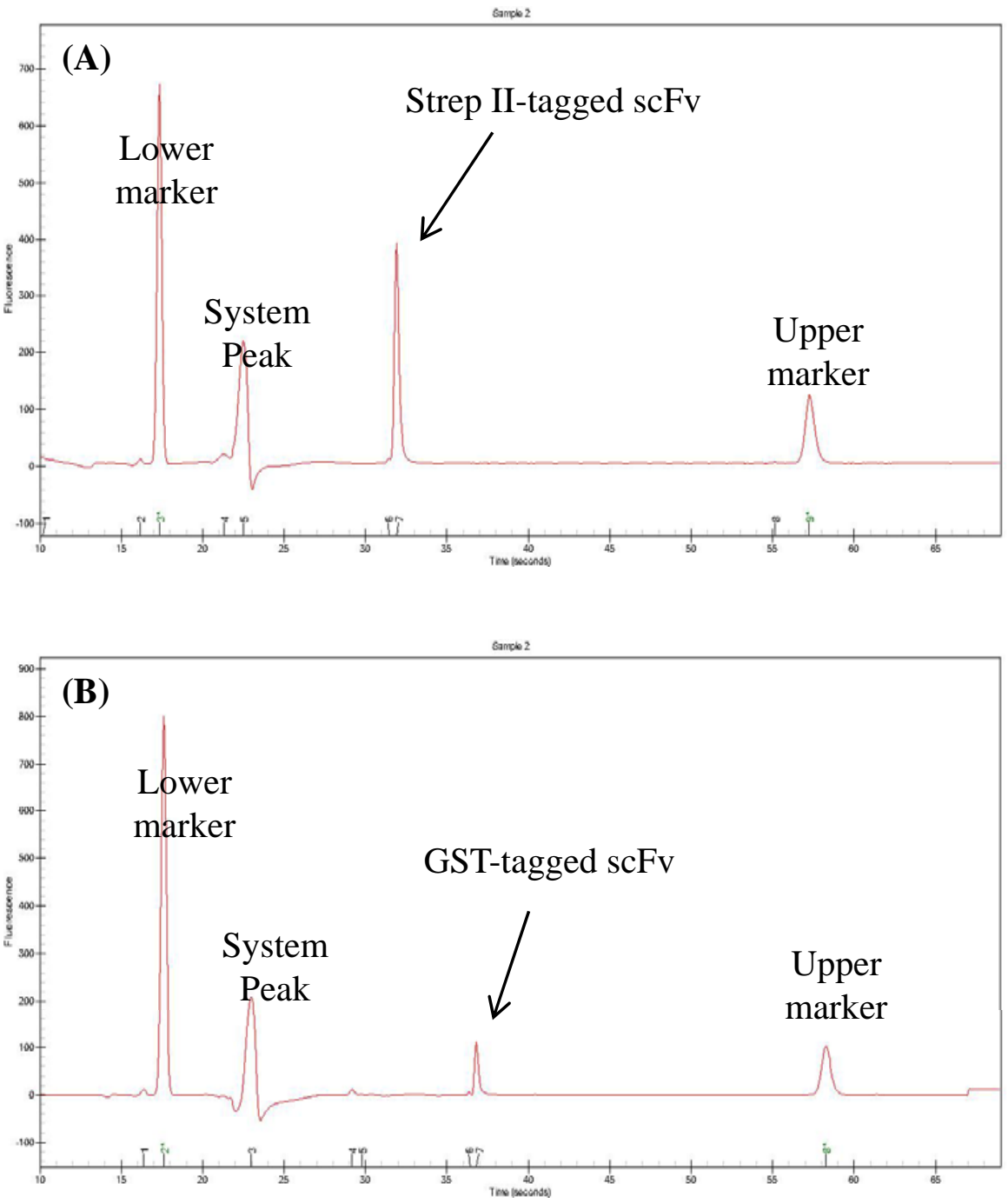